\title{
Correction: Billeaud et al. "Effects on Fatty Acid Metabolism of a New Powdered Human Milk Fortifier Containing Medium-Chain Triacylglycerols and Docosahexaenoic Acid in Preterm Infants" Nutrients 2018, 10, 690
}

\author{
Claude Billeaud ${ }^{1}$, Carole Boué-Vaysse ${ }^{2}$, Leslie Couëdelo ${ }^{2}{ }^{\circledR}$, Philippe Steenhout ${ }^{3}$, \\ Jonathan Jaeger ${ }^{4}$, Cristina Cruz-Hernandez ${ }^{4}$, Laurent Ameye ${ }^{5}$, Jacques Rigo ${ }^{6}$, \\ Jean-Charles Picaud ${ }^{7}{ }^{\circledR}$, Elie Saliba ${ }^{8}$, Nicholas P. Hays ${ }^{5}$ and Frédéric Destaillats ${ }^{5, *}$ \\ 1 CIC Pédiatrique 1401 CHU, 33000 Bordeaux, France; claude.billeaud@chu-bordeaux.fr \\ 2 ITERG, Université de Bordeaux, 33076 Bordeaux, France; c.vaysse@iterg.com (C.B.-V.); \\ 1.couedelo@iterg.com (L.C.) \\ 3 Nestlé Health Sciences, 1066 Epalinges, Switzerland; philippe.steenhout@nestle.com \\ 4 Nestlé Research Centre, 1000 Lausanne, Switzerland; jnthn.jaeger@gmail.com (J.J.); \\ cristina.cruz-hernandez@rdls.nestle.com (C.C.-H.) \\ 5 Nestlé Nutrition R\&D, 1800 Vevey, Switzerland; laurent.ameye@nestle.com (L.A.); \\ nicholaspaul.hays@nestle.com (N.P.H.) \\ 6 Department of Neonatology, University of Liège, 4000 Liège, Belgium; j.rigo@ulg.ac.be \\ 7 Hôpital de la Croix Rousse, Hospices Civils, 69004 Lyon, France; jean-charles.picaud@chu-lyon.fr \\ 8 Hôpital Clocheville, CHU de Tours, 37004 Tours, France; elie.saliba@univ-tours.fr \\ * Correspondence: frederic.destaillats@nestle.com; Tel.: +41-21-924-6217
}

Received: 6 March 2019; Accepted: 7 March 2019; Published: 22 March 2019

check for updates

The authors wish to make a correction to the published version of their paper [1]. While replying to a Letter to the Editor [2], we re-examined our results and realized that an erroneous version of Table 4 had been accidentally incorporated into the paper. During the manuscript review process, we were asked to reorder the table columns, include the standard deviations, and include all of the $p$ values. Due to the substantial nature of these edits, many errors were accidentally introduced into this table. We sincerely apologize for this unfortunate error and regret any subsequent confusion. A corrected version of Table 4 is below.

Table 4. (Corrected) Fatty acid profile (g/100 g of fatty acids) of total plasma phospholipids in preterm infants, receiving human milk fortified with a control (cHMF) or with a new human milk fortifier (nHMF) before and after 21 days of treatment. Estimates of the treatment effect nHMF/cHMF (difference) and the two-sided $p$-values are given for each fatty acid analyzed in the different lipid compartments.

\begin{tabular}{|c|c|c|c|c|c|c|c|c|c|c|}
\hline & \multicolumn{4}{|c|}{ cHMF $(n=21)$} & \multicolumn{4}{|c|}{ nHMF $(n=26)$} & \multirow{3}{*}{ Difference } & \multirow{3}{*}{$p$ Value } \\
\hline & \multicolumn{2}{|c|}{ Baseline } & \multicolumn{2}{|c|}{ After 21 Days } & \multicolumn{2}{|c|}{ Baseline } & \multicolumn{2}{|c|}{ After 21 Days } & & \\
\hline & Mean & SD & Mean & SD & Mean & SD & Mean & SD & & \\
\hline $14: 0$ & 0.21 & 0.14 & 0.22 & 0.14 & 0.24 & 0.13 & 0.26 & 0.13 & 0.184 & 0.500 \\
\hline $15: 0$ & 0.13 & 0.10 & 0.18 & 0.26 & 0.14 & 0.08 & 0.12 & 0.03 & -0.071 & 0.722 \\
\hline 16:0 & 25.00 & 5.55 & 22.75 & 6.21 & 26.00 & 3.58 & 26.46 & 2.76 & 0.180 & 0.020 \\
\hline 16:0 DMA & 0.63 & 0.18 & 0.70 & 0.17 & 0.64 & 0.16 & 0.74 & 0.27 & -0.134 & 0.394 \\
\hline $16: 1 n-7$ & 1.24 & 0.71 & 1.05 & 1.19 & 1.28 & 0.69 & 0.93 & 0.70 & 0.209 & 0.188 \\
\hline $16: 1 n-9$ & 0.32 & 0.14 & 0.24 & 0.15 & 0.30 & 0.12 & 0.25 & 0.13 & 0.219 & 0.117 \\
\hline 18:0 & 14.99 & 2.90 & 17.25 & 4.15 & 14.51 & 1.65 & 15.84 & 1.63 & -0.060 & 0.203 \\
\hline 18:0 DMA & 0.33 & 0.12 & 0.47 & 0.37 & 0.35 & 0.10 & 0.36 & 0.14 & -0.402 & 0.040 \\
\hline
\end{tabular}


Table 4. Cont.

\begin{tabular}{|c|c|c|c|c|c|c|c|c|c|c|}
\hline & \multicolumn{4}{|c|}{ cHMF $(n=21)$} & \multicolumn{4}{|c|}{ nHMF $(n=26)$} & \multirow{3}{*}{ Difference } & \multirow{3}{*}{$p$ Value } \\
\hline & \multicolumn{2}{|c|}{ Baseline } & \multicolumn{2}{|c|}{ After 21 Days } & \multicolumn{2}{|c|}{ Baseline } & \multicolumn{2}{|c|}{ After 21 Days } & & \\
\hline & Mean & SD & Mean & SD & Mean & SD & Mean & SD & & \\
\hline 18:1 DMA & 0.29 & 0.14 & 0.32 & 0.11 & 0.30 & 0.09 & 0.30 & 0.13 & -0.288 & 0.054 \\
\hline $18: 1 n-7$ & 3.07 & 0.59 & 2.36 & 0.62 & 3.05 & $0 ; 89$ & 2.59 & 0.77 & 0.122 & 0.015 \\
\hline $18: 1 n-9$ & 14.04 & 2.16 & 11.98 & 3.37 & 13.90 & 3.03 & 12.45 & 2.97 & 0.051 & 0.286 \\
\hline trans-18:1 & 0.30 & 0.15 & 0.42 & 0.16 & 0.39 & 0.13 & 0.40 & 0.17 & -0.112 & 0.515 \\
\hline $18: 2 n-6(\mathrm{LA})$ & 12.90 & 3.20 & 14.60 & 2.53 & 14.03 & 2.75 & 14.69 & 2.55 & 0.043 & 0.452 \\
\hline $18: 3 n-3$ (ALA) & 0.18 & 0.14 & 0.11 & 0.05 & 0.18 & 0.10 & 0.17 & 0.08 & 0.219 & 0.125 \\
\hline 18:3 n-6 (GLA) & 0.22 & 0.07 & 0.17 & 0.06 & 0.22 & 0.06 & 0.16 & 0.04 & 0.030 & 0.689 \\
\hline $20: 0$ & 0.36 & 0.29 & 0.40 & 0.31 & 0.38 & 0.21 & 0.41 & 0.18 & 0.011 & 0.945 \\
\hline $20: 1 n-9$ & 0.25 & 0.08 & 0.31 & 0.09 & 0.27 & 0.10 & 0.36 & 0.12 & 0.156 & 0.072 \\
\hline $20: 2 n-6$ & 0.47 & 0.13 & 0.67 & 0.46 & 0.51 & 0.11 & 0.53 & 0.10 & -0.112 & 0.340 \\
\hline $20: 3 n-6$ (DGLA) & 3.79 & 0.89 & 4.04 & 1.05 & 3.46 & 0.72 & 3.45 & 0.68 & -0.161 & 0.041 \\
\hline $20: 3 n-9$ & 2.56 & 1.46 & 1.90 & 1.53 & 2.12 & 1.19 & 2.11 & 1.51 & 0.089 & 0.612 \\
\hline $20: 4 n-6$ (ARA) & 9.81 & 2.33 & 10.63 & 3.54 & 9.09 & 2.03 & 8.26 & 2.58 & -0.211 & 0.015 \\
\hline $20: 5 n-3$ (EPA) & 0.84 & 0.48 & 0.70 & 0.25 & 0.88 & 0.32 & 0.97 & 0.28 & 0.318 & 0.006 \\
\hline $22: 0$ & 0.46 & 0.39 & 0.53 & 0.53 & 0.53 & 0.33 & 0.57 & 0.27 & 0.110 & 0.567 \\
\hline $22: 1 n-9$ & 0.05 & 0.03 & 0.05 & 0.03 & 0.05 & 0.02 & 0.05 & 0.02 & 0.048 & 0.676 \\
\hline $22: 4 n-6$ & 0.46 & 0.17 & 0.49 & 0.29 & 0.40 & 0.13 & 0.35 & 0.10 & -0.257 & 0.032 \\
\hline $22: 5 n-3$ ( $n-3$ DPA $)$ & 0.46 & 0.24 & 0.53 & 0.31 & 0.44 & 0.12 & 0.40 & 0.12 & -0.217 & 0.037 \\
\hline $22: 5 n-6(n-6$ DPA $)$ & 0.49 & 0.19 & 0.53 & 0.25 & 0.45 & 0.25 & 0.50 & 0.22 & -0.005 & 0.970 \\
\hline $22: 6 n-3$ (DHA) & 3.60 & 1.42 & 3.96 & 1.94 & 3.22 & 0.93 & 3.54 & 1.04 & -0.050 & 0.575 \\
\hline $24: 0$ & 0.39 & 0.29 & 0.53 & 0.80 & 0.48 & 0.28 & 0.43 & 0.18 & -0.056 & 0.742 \\
\hline $24: 1 n-9$ & 1.50 & 1.23 & 1.47 & 0.83 & 1.55 & 0.98 & 1.77 & 0.73 & 0.191 & 0.368 \\
\hline
\end{tabular}

Data are presented as geometric mean and geometric standard deviation (SD). ALA, $\alpha$-linolenic acid; ARA, arachidonic acid; DGLA, dihomo- $\gamma$-linolenic acid; DHA, docosahexaenoic acid; DMA, dimethyl acetal; DPA, docosapentaenoic acid; EPA, eicosapentaenoic acid; GLA, $\gamma$-linolenic acid; LA, linoleic acid.

This change does not impact the overall results or scientific conclusions. The original manuscript will remain online on the article webpage, with a reference to this correction.

\section{References}

1. Billeaud, C.; Boué-Vaysse, C.; Couëdelo, L.; Steenhout, P.; Jaeger, J.; Cruz-Hernandez, C.; Ameye, L.; Rigo, J.; Picaud, J.C.; Saliba, E.; et al. Effects on fatty acid metabolism of a new powdered human milk fortifier containing medium-chain triacylglycerols and docosahexaenoic acid in preterm infants. Nutrients 2018, 10, 690. [CrossRef] [PubMed]

2. Bernhard, W.; Poets, C.F.; Franz, A.R. Letter to the Editor Re: Billeaud et al. Nutrients 2018, 10, 690. Nutrients 2019, 11, 103. [CrossRef] [PubMed]

(C) 2019 by the authors. Licensee MDPI, Basel, Switzerland. This article is an open access article distributed under the terms and conditions of the Creative Commons Attribution (CC BY) license (http://creativecommons.org/licenses/by/4.0/). 